\title{
COMMUNICATION
}

Cite this: DOI: 10.1039/xoxxooooox

Received 8th January 2015,
Accepted ooth January 2015

DOI: 10.1039/xoxx00000x

www.rsc.org/chemcomm

\section{Electronic-Structure-Based Material Descriptors: (In)dependence on Self-Interaction and Hartree-Fock Exchange}

\author{
A. Notario-Estévez, S. M. Kozlov, F. Viñes ${ }^{*}$ and F. Illas
}

\begin{abstract}
Rational design of improved transition metal based materials mostly relies on their electronic structure descriptors, typically estimated by density functional theory and so unduly affected by self-interaction or static correlation errors. Here we show for all 30 transition metals that original or width-corrected $d$-band center, and Hilbert transform highest peak descriptors are unaffected by self-interaction, while poor treatment of static correlation by hybrids functionals leads to an unbalanced description. Thus descriptors have a general validity unbiased by a specific computational method.
\end{abstract}

The interaction of atoms and molecules with solid surfaces is the mainstay supporting many technologically relevant research areas: From surface and materials science, to tribology, nanotechnology, and eventually, heterogeneous catalysis. Solely the molecular adsorption on solid surfaces is of utmost importance, when related e.g. to sequestration of greenhouse effect gases, ${ }^{1}$ acid rain precursors, ${ }^{2}$ hydrogen storage for fuel cells, ${ }^{3}$ and molecular spintronics. $^{4}$ Self-assembled monolayers or molecular adlayers following a driven pattern can even lend the surface with selected properties; chirality or a magnetic moment, to name a few. ${ }^{5,6}$ In turn, molecular bond breakage/creation surface phenomena are crucial in heterogeneous catalysis. Most important large-scale chemical processes are heterogeneously catalyzed using scarce materials such as precious coinage and Pt-group transition metals. The optimization of the adsorptive or catalytic power of transition metal based materials is of paramount importance in the quest towards enhanced or fine-tuned chemical activities, moreover vital for an economic and energetic sustainable future.

The bonding between an adsorbate and a transition metal surface is inherently governed by the quantum interaction between their orbitals and bands, respectively. Thus, the rational optimization has been tackled using electronic structure terms. ${ }^{7}$ In particular, the Newns-Anderson model allowed for predicting the adsorbate-surface bond strength within the framework of perturbation theory. It revealed the pivotal effect of metal substrate Density Of States (DOS) shape on the nature of its chemical binding with adsorbates. ${ }^{8,9}$ For transition metals, the adsorbate interaction with half-filled broad $s$-band is considered constant due to the similar shape for all transition metals. Therefore, the interaction of adsorbate electron states with narrower $d$-band is alleged to govern chemical activity trends along transition metals.

The mathematical complexity of Newns-Anderson model as well as its explicit dependency on the adsorbate electronic structure limited its practical application. Instead, various parameters of substrate electronic structure were used as descriptors for different properties of interest, such as adsorption energies and reaction energy barriers. Presently these descriptors constitute indeed the only rational way of posing new transition metal based materials with adsorptive/reactive properties tuned at will, which is at the heart of heterogeneous catalysis. These descriptors are most often based in the so-called $d$-band model, which is discussed and justified in due detail in Ref. 10. Note here that as a consequence the $d$-band center, $\varepsilon_{\mathrm{d}}$, is used as a simple yet effective descriptor. The $\varepsilon_{\mathrm{d}}$ is normally referred to the Fermi energy $\varepsilon_{\mathrm{F}}$, see Fig. 1, and calculated as the gravimetric center or first moment of the $d$-Projected DOS (PDOS). ${ }^{11,12}$ This descriptor has been tremendously successful in predicting adsorptive and reactive properties as a function of structural saturation, strain, and alloy composition, ${ }^{6,13}$ including surface and subsurface alloys. ${ }^{14-17}$ However, in a few cases, the $d$ band center descriptor has been found to be inadequate for a few cases, ${ }^{18,19}$ and to loose accuracy on late transition metals, ${ }^{20}$ for instance.

Recent studies addressed the improvement of first-order $d$-band center descriptor. In particular, the poorer alignment of late transition metals has been attributed to the proportionality deviations of $d$-band width $W,{ }^{21}$ mainly affecting latest transition metals. Thus, a width-corrected descriptor $\varepsilon_{\mathrm{d}}{ }^{\mathrm{W}}=\varepsilon_{\mathrm{d}}+{ }^{W} / 2$ has been suggested and validated very recently, allowing for a better description of late transition metals without losing the accuracy of the earlier ones. Furthermore, accounting for $d$-band shape has been suggested via using as a descriptor the upper $d$-band edge, $\varepsilon_{\mathrm{u}}$, defined as the highest peak position of the Hilbert transform on the $d$-PDOS. ${ }^{22}$ This latter descriptor has been found to be a better reactivity descriptor for late transition metals and their alloys. This settled these descriptors as a predictive powerful tool backed up by the discovered trends. 


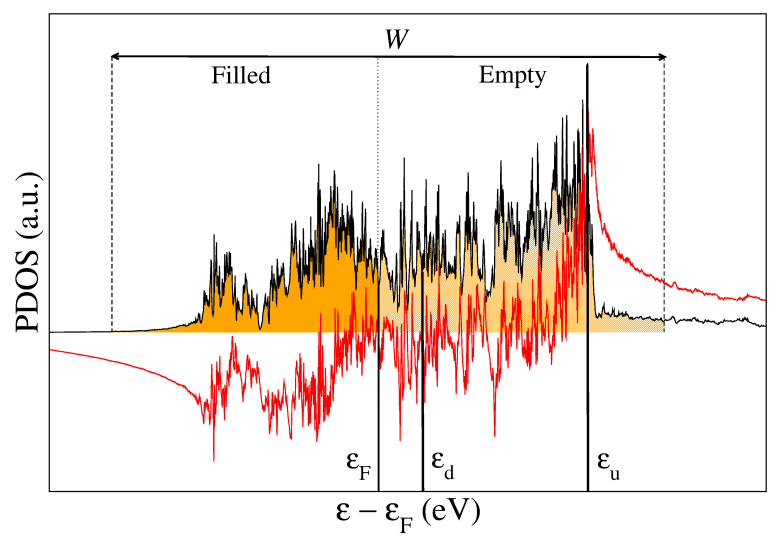

Fig. 1 Sketch of a $d$-band (black line) as obtained from a PDOS and its Hillbert transform (red line). Electronic-structure-based descriptors and parameters such as gravimetric center of the $d$-band, $\varepsilon_{\mathrm{d}}$, the band width, $W$, and the highest peak position of the Hillbert transform, $\varepsilon_{\mathrm{u}}$, are shown. Energy values $\varepsilon$ are referred to Fermi energy, $\varepsilon_{\mathrm{F}}$. The occupied $d$-states are shown by dark orange filling, unoccupied - light orange.

All these electronic-structure-based descriptors though strongly rely on the correct description of the electronic structure, i.e. the (P)DOS, including both occupied and virtual states. These DOS are often obtained using various approximations to Density Functional Theory $(D F T)$, which incorporate inherent deficiencies, such as the self-interaction error. The Local Spin Density Approximation $(L S D A)$ and Generalized Gradient Approximation (GGA) approximations are the first and second rungs, respectively, of Jacob's ladder of exchange-correlation $(x c)$ functionals, and exhibit the largest self-interaction error. ${ }^{23}$ An improvement is achieved with meta-GGA functionals, which constitute the third rung by further adding the kinetic energy density. Meta-GGA $x c$ functionals have the potential to reduce self-interaction error, ${ }^{24}$ although seldom represent an improvement to GGA in solid metals, as they do for molecular systems. ${ }^{25}$ In general, all these $x c$ functionals yield inaccurate, even incorrect description of the electronic structure of solids seen, for example, in band gaps of many transition metal oxides, ubiquitous as supports in catalysis, photocatalysis, and nanotechnology.

Finally, a good behavior was found in molecular chemistry ${ }^{26,27}$ by redeeming the $x c$ functionals through inclusion of Hartree-Fock $(H F)$ exchange in their ansatz. This constitutes the fourth rung of the ladder. However, for solid systems, and in particular transition metals, Jacob's ladder gets slippery at the top. The addition of HF exchange in extended systems increases the computational demands, and amplifies the error caused by imperfect treatment of static correlation, particularly important in transition metal compounds. In fact, HF exchange leads to an unphysical DOS of metals with a singularity in the electron group velocity at Fermi level, although this defect can be remediated if HF exchange is screened by correlation effects. ${ }^{28}$ In summary, the inclusion of HF exchange is detrimental for the accuracy of $x c$ functionals in bulk transition metals, due to the itinerant character of electrons.

Consequently, the electronic structure of a transition metal based system appears to be critically affected by the employed $x c$ functional. Hence, the optimization of the adsorptive and catalytic power of a material constituted by transition metals in terms of electronic structure based descriptors seems to be a matter of choice of the $x c$ functional, or does it not?

To investigate this we firstly obtained the DOS and $d$ PDOS of all $3 d, 4 d$, and $5 d$ transition metals using LSDA Vosko-Wilk-Nussair
$(V W N)$, GGA Perdew-Burke-Ernzerhof $(P B E)$, meta-GGA TaoPerdew-Staroverov-Scuseria (TPSS), and hybrid Heyd-ScuseriaErnzerhof (HSE06) xc functionals (see computational details in Supplementary Information). These four $x c$ functionals represent, in average terms, the most accurate ones for each of Jacob's ladder rungs in the description of structural, energetic, and elastic properties of transition metal bulks ${ }^{29,30}$ over 15 screened functionals. For each metal and each functional, the $\varepsilon_{\mathrm{d}}, \varepsilon_{\mathrm{d}}{ }^{\mathrm{W}}$, and $\varepsilon_{\mathrm{u}}$ descriptors have been gained (see computational details in Supplemental Information for further details of their obtaining, and Tables S1-S3 for a list of descriptor values).

A value comparison for the four encompassed $x c$ functionals is displayed in Fig. 2a-c. In these plots, PBE values are used in the abscissa axis, whereas the other $x c$ functional values are placed on the ordinate axis. Thus, PBE values feature an ideally linear trend, to which other $x c$ functionals are compared. Sign and color codes permit to discriminate in between $d$-series and $x c$ functionals. Let us begin the inspection on the $d$-band center $\varepsilon_{\mathrm{d}}$ descriptor, shown in Fig. 2a. For any $x c$ functional, values of a given $d$-series are scattered over the whole energy range, and no clustering of $d$-series is detected. For the LSDA, GGA, and meta-GGA rungs the $\varepsilon_{\mathrm{d}}$ values are oftentimes superimposed. The hybrid $x c$ behavior is discussed separately below. For these three first rungs it is clear that there are small deviations between local VWN and semi-local PBE and TPSS functionals, further supported by linear fit with regression coefficients above 0.997 , slopes close the ideal value of unity, and, finally, intercepts orbiting a zero value for VWN and TPSS.

An inspection of the DOS for different functionals on selected metals reveals slight discrepancies in the local shape of this function, although these differences are cancelled when computing the $\varepsilon_{\mathrm{d}}$ descriptor, as it hangs on the full $d$-band. The addition of a second central moment (i.e. width) correction to $d$-band center in $\varepsilon_{\mathrm{d}} \mathrm{W}$ descriptor, almost does not perturb the previous excellent agreement, see Fig. 2b. The slopes fluctuate near one, and the intercepts display just a slight negative value, anyhow, still close to zero. Moreover the regression coefficients are slightly worse, but still better than 0.993 .

So far it has been proven that for LSDA, GGA, and meta-GGA $x c$ functionals the utilization of one or another $x c$ functional does not affect $\varepsilon_{\mathrm{d}}$ and $\varepsilon_{\mathrm{d}}{ }^{\mathrm{W}}$ descriptors, i.e. they are not affected by the integration of the $d$-band nor its width. In Fig. 2c the effect of $x c$ functional components on $d$-band shape is put under the light, with outstanding results. It is remarkable to see that the highest peak of the Hillbert transform of the $d$-band, $\varepsilon_{\mathrm{u}}$, is essentially unaffected by the employed $x c$ functional. The obtained slopes for VWN and TPSS are near unity, and the intercepts essentially zero. The regression coefficients have excellent values better than 0.999 .

Care must be taken with hybrid functionals, as expected, due to their failure in properly describing the itinerant character of electrons in transition metal based compounds. ${ }^{28}$ Despite HSE06 $x c$ functional improves the correlation functional compared to other hybrid functionals, ${ }^{31}$ the deviations with respect to experiments are still notable. This is clear from the higher slope found for $\varepsilon_{\mathrm{d}}$ compared to PBE as shown in Fig. 2a, which is due to two additive factors. On one hand, the cancellation of self-interaction by the inclusion of HF exchange in HSE06 stabilizes the transition metal occupied bands, lowering their energy. On the other hand, the same origin has a diametrically opposed effect on the unoccupied region of the $d$-band, where bands become destabilized compared to PBE, similar to the effect on the conducting bands of transition metal oxides, see Fig. 3. This creates an asymmetric unbalance, which hangs upon the ratio of states on the occupied/unoccupied part of the $d$-band. Naturally, the unbalance is biased by the $d$-occupancy trend along a given transition metal series, with mild effect for those transition metals 
(a)

(b)
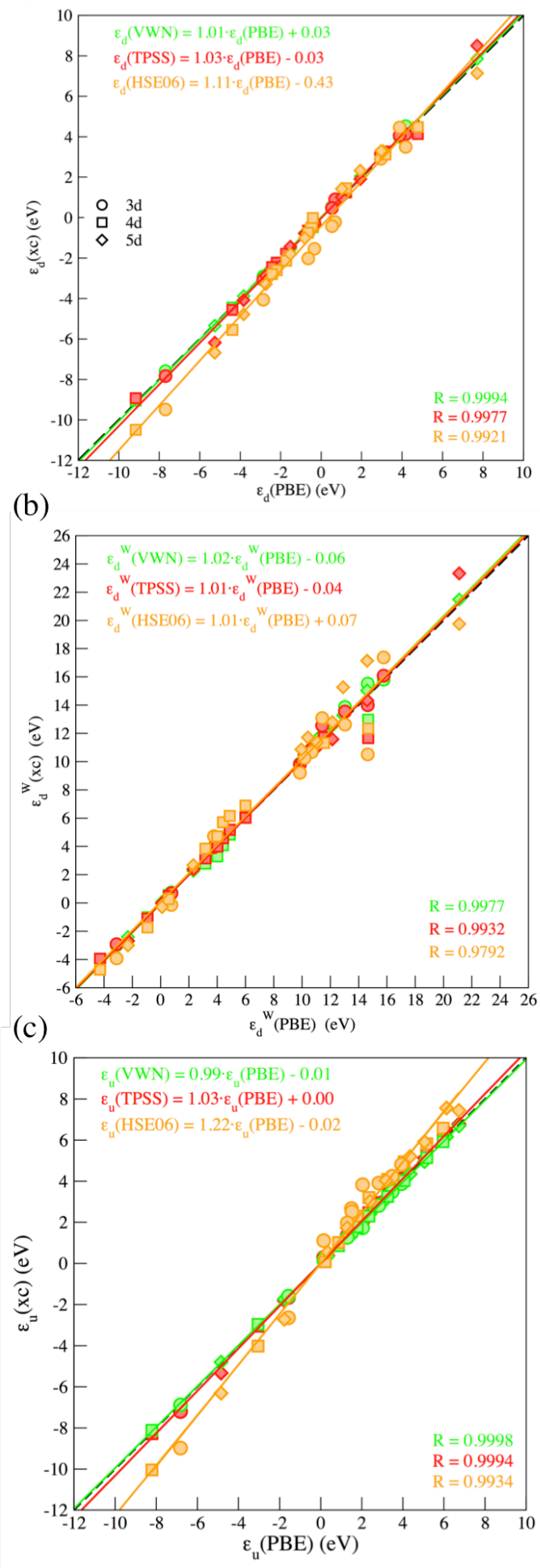

Fig. 2 Variation of (a) $d$-band center, $\varepsilon_{\mathrm{d}}$, (b) width-corrected $d$-band center, $\varepsilon_{\mathrm{d}}{ }^{\mathrm{W}}$, and (c) highest Hillbert transform peak, $\varepsilon_{\mathrm{u}}$, energy values calculated with a given $x c$ functional with respect to those obtained at PBE level, distinguishing between $3 d, 4 d$, and $5 d$ transition metal series using different symbols. Dashed black line represents ideal matching with respect PBE values. Colored solid lines belong to regressions of $x c$ values, whose slopes, intercepts, and regression coefficients, R, are displayed. whose $\varepsilon_{\mathrm{d}}$ lies near the $\varepsilon_{\mathrm{F}}$. The hybrid $x c$ usage also induces, in general, a band $W$ widening, as shown in Fig. 3. However, for early transition metals, where self-interaction error is more critical, the linear inclusion of HF exchange leads to more localized $d$-orbitals.

Consequently, the overlap between $d$-orbitals decreases and the resulting $d$-band tightens. As a result, for medium and late transition metals, which display negative $\varepsilon_{\mathrm{d}}$ values, the band width correction moves HSE06 values closer to PBE ones. On the other hand, for early transition metals, with positive $\varepsilon_{\mathrm{d}}$ values, the mentioned band tightening does also sets HSE06 values closer to PBE ones. This, fortuitously, makes the slope of HSE06 data on Fig. 2b almost coincident to unity. Nevertheless, HSE0 $6 \varepsilon_{\mathrm{d}}{ }^{\mathrm{W}}$ values still present a sensible dispersion, highlighted in a poorer regression coefficient of 0.979. Last, in the case of Hillbert transform $\varepsilon_{u}$ descriptor, see Fig. $2 \mathrm{c}$, the highest peak seems to be affected likewise to the $d$-band center.

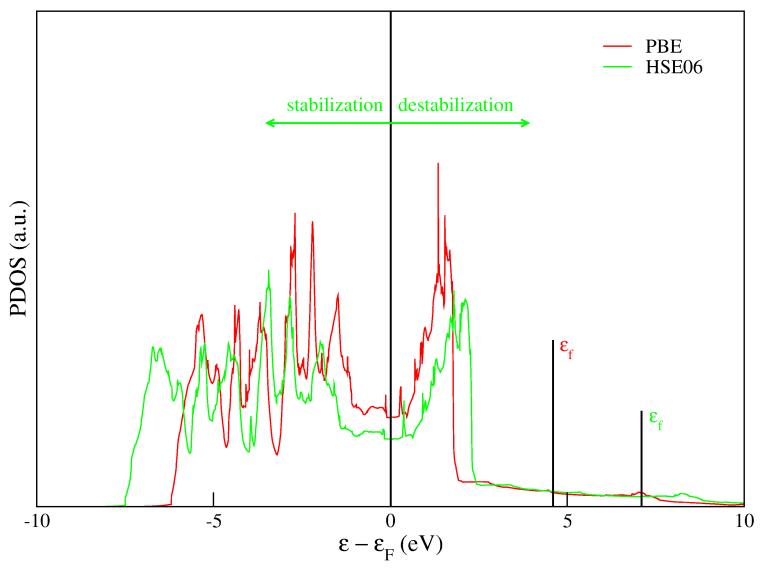

Fig. 3 The $d$-band PDOS of Ru as obtained at PBE and HSE06 xc functional levels, showing the stabilization and destabilization of occupied and unoccupied bands when incorporating HF exchange in HSE06. Solid black lines point the $d$-band end, $\varepsilon_{\mathrm{f}}$, for each functional.

In summary, the present work shows that transition metal $d$-band occupation, width, and shape do not depend on the particular choice of a given exchange-correlation potential, which implies that these properties are independent of the degree of self-interaction error introduced by each approximation of the $x c$ functional. Consequently, most popular descriptors, such as the $d$-band center $\varepsilon_{\mathrm{d}}$, the width corrected $d$-band center, $\varepsilon_{\mathrm{d}}{ }^{\mathrm{W}}$, and the highest peak of Hillbert transform, $\varepsilon_{\mathrm{u}}$, can be safely computed and even combined at any of first three Jacob's ladder rungs without losing their validity for the optimization of the activity of transition metal based materials of technological interest. Nonetheless, hybrid functionals are not advised when computing electronic structure based descriptors for transition metal materials.

This work was supported by Spanish Ministerio grants (CTQ201230751) and Generalitat de Catalunya grants (2014SGR97 and XRQTC). S. M. K. thanks the Spanish Ministerio de Educación for a predoctoral grant AP2009-3379. F. V. thanks the Spanish Ministerio de Economía y Competitividad for the Ramón y Cajal postdoctoral grant (RYC-2012-10129). F. I. acknowledges additional support through the ICREA Academia award for excellence in research. 


\section{Notes and references}

Departament de Química Física \& Institut de Química Teòrica $i$ Computacional (IQTCUB), Universitat de Barcelona, c/Martí i Franquès 1,08028Barcelona, Spain.E-mail: francesc.vines@ub.edu.

Electronic Supplementary Information (ESI) available: Computational details and details of how descriptor values are obtained, and Tables S1S3 provide a list of descriptor values for each metal and $x c$ functional used. See DOI: 10.1039/c000000x/

1 Y. X. Yang, J. Evans, J. A. Rodriguez, M. G. White and P. Liu, Phys. Chem. Chem. Phys., 2010, 12, 9909-9917.

2 J. A. Rodriguez, G. Liu, T. Jirsak, J. Hrbek, Z. P. Chang, J. Dvorak and A. Maiti, J. Am. Chem. Soc., 2002, 124, 5242-5250.

3 M. P. Suh, H. J. Park, T. K. Prasad and D. W. Lim, Chem. Rev., 2012, 112, 782-835.

4 S. Sanvito, Chem. Soc. Rev., 2011, 40, 3336-3355.

5 V. Humblot, S. Haq, C. Muryn, W. A. Hofer and R. Raval, J. Am. Chem. Soc., 2002, 124, 503-513.

6 M. Garnica, D. Stradi, S. Barja, F. Calleja, C. Días, M. Alcamí, N. Martín, A. L. Vázquez de Parga, F. Martín and R. Miranda, Nat. Phys., 2013, 9, 368-374.

7 Recently, a purely structural descriptor, the generalized coordination number, was also proposed in F. Calle-Vallejo, J. I. Martínez, J. M. García-Lastra, P. Sautet and D. Loffreda, Angew. Chem. Int. Ed., 2014, 53, 8316-8325. Although very useful, by construction it cannot grasp variations between different materials.

8 D. M. Newns, Phys. Rev., 1969, 178, 1123-1135.

9 P. W. Anderson, Phys. Rev., 1961, 124, 41-53.

10 B. Hammer and J. K. Nørskov, Surf. Sci., 1995, 343, 211-220.

11 T. Bligaard and J. K. Nørskov, Electrochim. Acta, 2007, 52, 55125516.

12 F. Abild-Pedersen, J. Greeley and J. K. Nørskov, Catal. Lett., 2005, 105, 9-13.

13 M. Mavrikakis, B. Hammer and J. K. Nørskov, Phys. Rev. Lett., 1998, 81, 2819-2822.

14 J. Greely and M. Mavrikakis, Nature Mat., 2004, 3, 810-815.

15 V. R. Stamenkovic, B. S. Mun, M. Arenz, K. J. J. Mayrhofer, C. A. Lucas, G. Wang, P. N. Ross and N.M. Markovic, Nature Mater., 2007, 6, 241-247.

16 L. A. Kibler, A. M. El-Aziz, R. Hoyer and D. M. Kolb, Angew. Chem. Int. Ed., 2005, 44, 2080-2084.

17 A. Ruban, B. Hammer, P. Stoltze, H. L. Skriver and J. K. Nørskov, J. Mol. Catal. A, 1997, 115, 421-429.

18 Z. P. Liu, S. J. Jenkins and D. A. King, J. Am. Chem. Soc., 2004, 126, 10746-10756.

19 T. Hofmann, T. H. Yu, M. Folse, L. Weinhardt, M. Bär, Y. Zhang, B. V. Merinov, D. J. Myers, W. A. Goddard III and C. Heske, J. Phys. Chem. C, 2012, 116, 24016-24026.

20 T. H. Yu, T. Hofmann, Y. Sha, B. V. Merinov, D. J. Myers, C. Heske and W. A. Goddard III, J. Phys. Chem. C, 2013, 17, 26598-26607.

21 A. Vojvodic, J. K. Nørskov and F. Abild-Pedersen, Top. Catal., 2014, 57, 25-32.

22 H. Xin, A. Vojvodic, J. Voss, J. K. Nørskov and F. Abild-Pedersen, Phys. Rev. B, 2014, 89, 115114-115118.

23 J. P. Perdew and K. Schmidt, AIP Conf. Proc., 2001, 577, 1-20.
24 A. D. Becke, J. Chem. Phys., 1998, 109, 2092-2098.

25 K. Yang, J. Zheng, Y. Zhao and D. G. Truhlar, J. Chem. Phys., 2010, 132, 164117.

26 S. F. Sousa, P. A. Fernandes and M. J. Ramos, J. Phys. Chem. A, 2007, 111, 10439-10452.

27 R. Peverati and D. G. Truhlar, Phys. Trans. Roy. Soc. A, 2014, 372, 20120476.

28 N. W. Ashcroft and N. D. Mermin, Solid State Physics, Saunders College Publishing, Orlando, 1976.

29 P. Janthon, S. M. Kozlov, F. Viñes, J. Limtrakul and F. Illas, J. Chem. Theory Comput., 2013, 9, 1631-1640.

30 P. Janthon, S. Luo, S. M. Kozlov, F. Viñes, J. Limtrakul, D. G. Truhlar and F. Illas, J. Chem. Theory Comput., 2014, 10, 3832-3839.

31 J. Paier, M. Marsman and G. Kresse, J. Chem. Phys., 2007, 127, 024103-024112. 